\title{
Time-resolved optical-UV spectroscopy of colliding wind effects
}

\author{
Anthony F.J. Moffat ${ }^{1}$ \\ Département de physique, Université de Montréal, \\ C.P. 6128, Succ. Centre-Ville, Montréal, Qc H3C 3J7, Canada
}

\begin{abstract}
It is in the ultraviolet-optical domain where the strongest known emission lines arise in hot star winds. In the case of hot-star binaries, culminating in the relatively common, strong-wind $\mathrm{WR}+\mathrm{O}$ systems, similar line-emission is seen in the cooling flows downstream from the highly compressed, X-ray emitting heads of the bow shock regions produced when the two winds collide. Timeresolved UV-optical spectroscopy of these flows around a complete orbit can provide important constraints not only on the colliding wind process itself, but also on the winds and the orbit. Spectroscopic wind-wind collision effects have now been seen in every relatively close WR+O system $(P \lesssim 100 \mathrm{~d})$ that has been adequately observed so far.
\end{abstract}

\section{Introduction}

Massive stars are notorious for their strong winds, which are also fast if the stars are hot. In the case of single massive hot stars, the winds will collide first with the ISM, then with each other as fast-wind phases catch up with preceeding slower winds as the underlying star crosses the H-R diagram from cool back to hot. These interactions often lead to the so-called 'ring nebulae', on scales of the order of a parsec.

In the case of massive binaries containing hot stars (mainly $\mathrm{O}$ and WR type), the winds can collide on a scale determined by the orbital separation: 10 s of solar radii for the closest systems to 100 s of $\mathrm{AU}$ for the widest observed systems (see below). Among the possible combinations of different stellar types in hot-star binaries, those systems containing a WR and an $\mathrm{O}$ star appear to release the most energy in a shock zone created by wind-wind collision (WWC). The effects are weaker and more difficult to quantify in $\mathrm{O}+\mathrm{O}$ systems (e.g., Gies 1996), while WR+WR systems are extremely rare, if any exist at all. Hence, I will restrict the remaining part of this review to $\mathrm{WR}+\mathrm{O}$ systems, for which considerable interest has arisen in very recent years.

\section{Evidence}

Although not really 'evidence' per se, theoretical calculations show that WWC shock cones should occur when two smooth, spherically symmetric hot-star

\footnotetext{
${ }^{1}$ Killam Fellow of the Canada Council for the Arts
} 
winds collide (e.g., Stevens et al. 1992 for numerical work; Eichler \& Usov 1993 for analytical work). In particular, these studies show that, while X-ray temperatures can occur in the region of the bow shock head where the winds collide $\sim$ head-on, downstream from this rapid cooling can occur along a nearly linear shock cone of half-opening angle $\Theta$ and angular extent $\Delta \Theta$. To within a degree, the former can be expressed in terms of the ratio of the terminal wind momentum transfer rates $\eta=\left(\dot{M} v_{\infty}\right)_{\mathrm{O}} /\left(\dot{M} v_{\infty}\right)_{\text {WR }}$ (or what turns out to be the squared ratio of distances from the centre of each star to the stagnation point between the stars, $\eta=\left(r_{\mathrm{O}} / r_{\mathrm{WR}}\right)^{2}, \Theta=120^{\circ}\left(1-\eta^{2 / 5} / 4\right) \eta^{1 / 3}$. Note that for $\eta=0$ and 1 (extreme limits), $\Theta=0^{\circ}$ and $90^{\circ}$, respectively, i.e., a pencil-sharp shockcone for highly dominating WR wind, to a contact surface defined by a plane for equal winds. One also has $\Delta \Theta \approx \Theta$ for an adiabatic flow, while $\Delta \Theta \ll \Theta$ for efficient cooling (e.g., for close systems where the WWC zone density is high).

The real, hard evidence is in the observations. These can be sorted into two classes: resolved and unresolved WWC zones. The former is the hardest evidence, and has been only seen in several rare cases so far: from (nonthermal) radio images of the bow head region of two wide visual binaries WR 146, WC6+O (Dougherty et al. 1996) and WR 147, WN8+OB (Williams et al. 1997), and in near-IR emission from dust in the 13.1-year WC7+OB binary WR 137 (Marchenko, Moffat \& Grosdidier 1999a and in these Proceedings). In all three cases, the emitting regions are highly clumped.

As for unresolved evidence, there is plenty. This includes excess non-thermal radio emission, variable $\mathrm{X}$-ray emission, and variable IR emission from dust forming in the WWC zone (see other reviews of colliding winds in these Proceedings). It also includes excess spectral-line emission and associated phasedependent line-profile variations (LPVs) arising downstream in the WWC zone. This latter is the subject of this review.

\section{Observations of WWC line emission: general}

\subsection{Lines versus continuum}

It is useful to contrast what one can obtain from spectral lines versus continuum emission arising in the WWC zone (unresolved). While non-thermal radio and $\mathrm{X}$-ray continuous emission is generated in the hot bow head, IR continuous emission arises far downstream along the shock cone, from hot, clumped dust. The dust has formed via ram compression of the gas and shielding from the lethal UV radiation fields of the stars, aided by extreme clumping, as is now indeed seen in WR 137.

Excess line emission in the UV, optical and (near-)IR that is observed from the WWC zone, must arise between the hot bow head and the cooler dust forming region. The advantage of lines is that one has Doppler information, that (unresolved) continuous emission does not offer. From this, we can deduce geometrical parameters of the WWC collision process that cannot be done (as easily and directly) from the continous emission. However, it should be noted that the powerful diagnostic of X-ray line emission is starting to be exploited, and nothing can really beat being able to resolve the WWC zone directly. The latter will become possible once ultra-high resolution optical/IR interferometry becomes a reality. 
Unfortunately though, excess line emission from the WWC zone often coincides with similar line emission from the WR wind, which can vary in different ways (e.g., Marchenko et al. 1997). These include orbital radial velocity (RV) changes, disk eclipses, atmospheric eclipses, heating effects and gravitational distortions, all of which vary with orbital phase. The first three require relatively high orbital inclination, $i$. To these effects, one can add corotating interactive regions, clumps, variable global mass-loss rate and possibly other heretofore unrecognized effects that can affect the line emission without being correlated with orbital phase.

\subsection{Emission versus absorption lines}

Another interesting distinction to make is between emission- and absorptionlines arising in WWC zones. As will be discussed below, emission excess from WWC zones allows a global view at all orbital phases and any inclination. Most cases are optically thin, so that the equivalent width of the excess emission $\left(W_{e}\right)$ remains $\sim$ constant with phase. However, the profile shape can change as a function of phase, allowing one to determine various parameters relating to the geometry and flow speed (see below). On the other hand, absorption through the WWC excess density requires back illumination, so its visibility is limited to special phases, normally $\sim 0.6 \pm 0.1$, i.e., just after passage of the O-star in front for a circular orbit, and $i>90^{\circ}-\Theta(e . g$. , the optical work on V444 Cyg: Marchenko et al. 1997).

\subsection{Optical versus ultraviolet lines}

Yet another distinction can be made between optical (including in principle also near-IR lines) and UV lines. While the UV contains mostly resonance and low-excitation lines formed in more remote areas, the optical generally contains weaker, subordinate lines formed closer to the source. This makes the optical more effective to trace the WWC zone globally in optically thin emission lines. Furthermore, WWC effects can often be confused in the UV with selective wind eclipses (e.g., St-Louis 1995). Most UV spectroscopy has been done using IUE, hence of limited S/N (typically 20-30), in contrast with the optical, where S/N reaching 300 and more can be obtained easily. On the other hand, the availability of resonance lines in the UV allows one better to estimate the terminal velocities of both stars (e.g., V444 Cyg: Shore \& Brown 1988). Given all its advantages, we will thus concentrate on optical observations in the rest of this review.

\section{Observations of WWC line emission: optical}

\subsection{Case studies}

Table 1 containes a list of $23 \mathrm{WR}+\mathrm{O}$ binaries in which the effects of WWC have been sought and explicitly reported. It includes 18 Galactic, 3 LMC and 2 SMC systems. In the Galaxy, at least 10 more systems not included in Table 1 are within easy reach, while in the Magellanic Clouds (MCs), a programme is in progress by Foellmi et al. (1999) to examine all known WN spectra in the SMC and all known WNE spectra in the LMC for binaries; with subsequent study of 
Table 1. WR+O binaries spectroscopically sought for WWC

\begin{tabular}{llrlll}
\hline & & & & \\
star & spectrum & $P(\mathrm{~d})$ & WWC? & remarks & references \\
& & & & & \\
WR 9 & WC4+O7 & 14.3 & strong & & 1 \\
WR 11 & WC8+O9 & 78.5 & moderate & & 2,3 \\
WR 21 & WN50+O5 & 8.3 & moderate & & 4,5 \\
WR 22 & WN7ha+O9 & 80.4 & weak & eclipsing & 6,7 \\
WR 30a & WO5+O4 & 4.6 & moderate & & 1 \\
WR 42 & WC7+O7V & 7.9 & strong & & 8 \\
WR 46 & WN3bpec & 0.3 & (v. strong) & WR+c? & 9,10 \\
WR 48 & WC6[+O9.5I] & 18.3 & v. strong & & $4,11,12$ \\
WR 79 & WC7+O5-8 & 8.9 & strong & & $4,8,13$ \\
WR 113 & WC8+O8-9IV & 29.7 & strong & (eclipsing) & 14 \\
WR 133 & WN50+O9I & 112.8 & weak & & 15 \\
WR 137 & WC7+OB & $13.1 y r$ & no & & 16 \\
WR 139 & WN50+O6 & 4.2 & strong & eclipsing & $5,17,18$ \\
WR 140 & WC7+O4-5 & $7.9 y r$ & & $e=0.85$ & \\
& & 170 & weak & equiv. $e=0$ & 19,20 \\
WR 141 & WN5o+O5 & 21.7 & v. weak & & 21 \\
WR 148 & WN8h & 4.3 & moderate & WR+c or B & 22 \\
WR 151 & WN40+O5V & 2.1 & strong & eclipsing & 23 \\
WR 155 & WN6o+O9 & 1.6 & moderate & eclipsing & 24 \\
Br 22 & WC4+O5-6 & 14.9 & strong & & 1 \\
Br 31 & WC4[+O8I:] & 3.0 & moderate & & 1 \\
Br 32 & WC4+O6 & 1.9 & moderate & & 1 \\
AB 5 & WNo+O7I: & 19.3 & v. strong & eclipsing & 25 \\
AB 8 & WO4+O7 & 16.6 & strong & & 1 \\
\hline & & & & & \\
\end{tabular}

Ref.: 1 - Bartzakos (1998), 2 - St-Louis et al. (1993), 3 - Eversberg et al. (1998), 4 - St-Louis et al. (1996), 5 - Koenigsberger \& Auer (1985), 6 - Raauw et al. (1996), 7 - St-Louis (1995), 8 - Hill et al. (1999a), 9 - Niemela et al. (1995), 10 - Veen et al. (1995), 11 - Moffat \& Seggewiss (1977), 12 - Hill et al. (1999b), 13 - Lührs (1997), 14 - Antokhin \& Moffat (1999), 15 - Bertrand (1995), 16 Moffat et al. (1999), 17 - Shore \& Brown (1988), 18 - Marchenko et al. (1997), 19 - Hervieux (1995), 20 - Setia-Gunawan et al. (1995), 21 - Marchenko et al. (1998), 22 - Marchenko et al. (1996), 23 - Lewis et al. (1993), 24 - Marchenko et al. (1995), 25 - Moffat et al. (1998).

Note: The shorter period for WR 140 corresponds to a circular orbit with component separation equal to the minimum separation of the observed highly elliptical orbit, all else being equal.

WWC effects, as did Bartzakos for the WC/WO stars in both MCs. Later, we will go after all the WNL stars to complete the LMC.

From Table 1, it is apparent that WWC LPVs are very common among $\mathrm{WR}+\mathrm{O}$ binaries, as long as the periods are not too long $(P \lesssim 100 \mathrm{~d})$. Main trends emerging are threefold: (i) WWC effects tend to be stronger in $\mathrm{WC}+\mathrm{O}$ systems than in WN+O systems; (ii) WWC effects are strongest for intermediate periods, in the range $\sim 5-30 \mathrm{~d}$; and (iii) the WWC spectrum tends to be later (cooler) in type than that of the WR star. The first is likely related to the enhanced cooling due to higher 'metal' abundance in WC stars compared to 
WN stars. The second is more or less as predicted by Eichler \& Usov (1993) allowing for radiative braking in short-period systems, and the third may be a consequence of the more rapid and effective cooling of the initially highly compressed gas in the WWC bow head.

Also noted are WWC effects seen in $\mathrm{O}+\mathrm{O}$ binaries (e.g., Gies 1996) as well as LBV binaries ( $\eta$ Car: Damineli et al. 1997 ; HD 5980 during eruption: Moffat et al. 1998). The former are more subtle than in WR+O binaries, due to the reduced wind density. $\eta$ Car is in a complex environment and is still being studied. During eruption in 1994, HD 5980 shows reduced WWC effects compared to its pre-eruption state, probably because the eruptor in this relatively short-period, elliptical system extended beyond the orbit of the (WR?) companion to the LBV.

\subsection{Analysis}

Two approaches to understand the LPVs of WWCs present themselves: numerical and analytic. The former involves hydrodynamical calculations of both winds and their interaction. Such was applied to observations of the near-IR He I line at $1.08 \mu$ in five WR+O binary systems by Stevens \& Howarth (1999). While potentially more complete, this approach is difficult for fitting parameters of the winds and the geometry of the interaction. While the results of Stevens \& Howarth are qualitatively interesting, they do not quantitatively fit the data well. They also assume that the pre-collision winds are smooth, which is strongly suspected not to be the case in virtually all hot-star winds (e.g., Eversberg et al. 1998).

While much simpler and approximate, the analytical approach is nevertheless quite powerful, because it allows one to fit parameters and understand better the underlying assumptions. One of the first attempts to deal with the problem of WWCs was the work of Wallerstein et al. (1984) in the context of symbiotic binaries. More recently, Lührs (1997) has devised an analytic model for the WWC zone in WR+O binaries, which I will elaborate on here ( $c f$. also Moffat 1998).

The Lührs' model first assumes optically thin, isotropic line emission from a section of an idealized, uniform, geometrically thin cone. The plausibility of such a cone is supported by hydrodynamic calculations of WWC for WR+O systems and illustrated, e.g., in Fig. 1 of Kallrath (1991), where the wall of the shock cone is nearly linear beyond the bow head. An exact form for the cone is given by Cantó et al. (1996); however, such a function will only complicate matters here, making the cone line-emission function non-integrable in closed form. The Lührs' model is applied to a circular orbit (for an elliptical orbit, cf. Moffat et al. 1998) of inclination $i$. The cone opening half-angle is $\Theta$ (as in the Eichler/Usov model) and the flow speed along the cone away from its hypothetical apex is designated by a constant value $v_{\text {strm }}$. In principle, different lines would be formed at different positions downstream along the cone: lines of high ionization formed closer to the source are expected to have slightly larger $\Theta$ and slightly smaller $v_{\text {strm }}$ than lines of lower ionization. For a given line transition, the emission is assumed to be uniform around the girth of the cone over the finite region where the line is formed.

Basically, the Lührs' model leads to a double-peaked emission-line profile, with cusps at the outer extremes and whose form changes with orbital phase for 
a given orbital inclination. This is not surprising in view of the even simpler well-known result for the simple case of a thin, symmetric, uniformly expanding and emitting ring ( $c f$. Moffat 1998). Even a uniformly rotating such ring gives the same type of line profile, with cusps at its two edges. The separation of the cusps corresponds to twice the expansion or rotation velocity, respectively, of the ring. Assumed to be optically thin, the Lührs' excess line emission flux remains constant with phase, as long as the emitting cone is not eclipsed. The excess emission can be simply characterized by its mean radial velocity (RV) and width (velocity separation between the cusps):

$$
\begin{gathered}
R V=v_{\text {strm }} \cos \Theta \sin i \cos (\phi-\delta \phi), \\
2 v_{\star}=2 v_{\text {strm }} \sin \Theta \sqrt{1-\sin ^{2} i \cos ^{2}(\phi-\delta \phi)},
\end{gathered}
$$

where $\phi$ is the orbital phase (WR star in front when zero) and $\delta \phi$ is the phase shift caused by the Coriolis effect of the binary motion. Note that for large values of $\delta \phi$, the emission from the cone surface will no longer by symmetric; it will be enhanced on the side closer to the WR star (e.g., Stevens \& Howarth 1999). However, it will be neglected here, assuming small values of $\delta \phi$. Note also the $90^{\circ}+\delta \phi$ phase lag of the shock cone mean emission RV compared to the orbit of the WR star, $v_{\text {orb }}(\mathrm{WR})=V_{\mathrm{o}}+K_{\mathrm{WR}} \sin \phi$.

Of course, we never actually see sharp cusps, even at high spectral resolution. The model therefore needs modification. One way to do this might be to replace the assumed constant streaming velocity by a distribution that allows for the turbulent nature of the WWC zone, as seen in numerical simulations. If this were isotropic, one could simply convolve the resulting line profile with a gaussian, whose width corresponds to the turbulent velocity. However, the velocity dispersion may in fact not be isotropic, as seen in stellar winds themselves, where the velocity dispersion is greater in the radial direction where the driving force occurs, than the azimuthal direction (Lépine \& Moffat 1999). If the same holds in WWC zones, the disperion may be greater outwards along the cone surface than perdendicular to it.

However, instead of this approach, Lührs modified his model to allow for finite width of the cone. To do this, he assumed that the $\Theta$-dependence of the WWC emission follows a parabolic law in $\cos \Theta$, starting at zero at the inner $\left(\Theta_{1}\right)$ and outer $\left(\Theta_{2}\right)$ edges of the cone and peaking at $\cos \Theta$ halfway between these two limits. This does indeed reduce the cusps to finite levels. Examples of Lührs' model profiles for different $i$ and $\Theta$ as a function of orbital phase are illustrated in Moffat (1996).

Besides not including turbulent broadening, the modified Lührs' model also does not allow for three other factors: (i) Sudden braking of the WR wind (Gayley et al. 1997) by the opposing radiation field of the O-star companion as the metal-rich WR wind approaches it. This effect will increase the cone opening angle $\Theta$ and tend to prevent crashing of the WR wind onto the O-star surface, for which there is little evidence in any case; (ii) The missing 'wedge' of the WR wind which is displaced by the shock cone and the O-star wind interior to it. Stevens \& Howarth (1999) attempted to model this latter factor, although with limited quantitative success. Possibly this factor is relatively small and can be mathematically 'absorbed' into the Lührs' cone emission function; and (iii) 
Asymmetry of the WWC profile, caused by Coriolis bending of the shock cone axis as a result of finite orbital motion relative to the wind. The side of the cone closest to the WR star is expected to emit more strongly as a result of higher density.

\subsection{Results}

Best results from applying the Lührs' model so far have been obtained by Hill et al. (1999a,b; and these Proceedings) for the two WC7+mid-O binaries WR 42 $(P=7.9 \mathrm{~d})$ and WR $79(P=8.9 \mathrm{~d})$. In particular and especially in WC stars, the C III 5696 line is most sensitive to density enhancements. This explains both the relatively large excess seen in this line (superposed on the relatively constant WR wind emission of the same transition), behaving at least qualitatively according to the Lührs' model, as illustrated in Hill et al. (1999a,b; these Proceedings) and St-Louis et al. (1996), both before and after extraction. Note how the excess emission exhibits its maximum redshift just after phase zero in both systems, when the O-star passes behind and the shock cone is pointing away from the observer. Of interest also is the small-scale variability of the WWC excess part of the profile, especially evident when comparing spectra at the same phase but different epochs. This is probably caused by the expected strong turbulence within the shock cone. The strong underlying WR emission in this line is also known to be highly variable in single WC stars, but at a relatively lower level than one appears to be seeing in the excess cone emission.

While one sees the clear (but variable) signature of double peaks in the excess emission in the broader-line star WR 79, such is much less obvious in WR 42. After fitting the Lührs' model, it appears that this is likely due to a combination of the greater proximity of the double peaks in WR 42 with turbulence and instrumental resolution. This may become more evident in new high-resolution spectral data recently obtained for these two stars by Lührs \& Moffat (1999). Variations in width of the WWC excess emission as expected from the Lührs' model also prevail, but are not so obvious upon visual inspection, especially for WR 42, which has very low orbital inclination and small cone opening angle. The resulting fits to the WWC excess (e.g., Fig. 1 of Hill et al., these Proceedings) appear to be quite good for WR 42, with no obvious systematic deviations at different phases. However for WR 79, there may be some lingering asymmetries not allowed for by the Lührs' model, e.g., excess emission in the blue peak around phases $0.3-0.6$ (the O-type star passes in front at phase $0.5)$. This might be the result of any of the shortcomings of the simple Lührs' model, as noted above.

The low values of the orbital inclination found for WR $42\left(23 \pm 6^{\circ}\right)$ and WR $79\left(33 \pm 2^{\circ}, c f .28^{\circ}\right.$ from Lührs 1997) by this technique are in fair agreement with other techniques. Values from polarisation, photometry and adopting a mass for the O-companion based on its spectral type (St-Louis et al. 1988; Lamontagne et al. 1996) are $\left(44^{\circ}, 40^{\circ}, 38^{\circ}\right)$ for WR 42 and $\left(45^{\circ}, 34^{\circ}, 34^{\circ}\right)$ for WR 79, with estimated uncertainties of several degrees in each case. A mean inclination of $36^{\circ}$ in each case converts the low values observed from the orbit of $M \sin ^{3} i$ to reasonable masses $\left(\sim 10\right.$ and $25 \mathrm{M}_{\odot}$ for the WR and $\mathrm{O}$ components, respectively).

In another study, Bartzakos (1998; see also Bartzakos et al. 1999) has completed a systematic search for binaries among all WC/WO stars in the 
Magellanic Clouds (MCs) resulting in only three WC4+O short-period binaries in the LMC and one WO4+O in the SMC. The WWC effects in these are compared to those seen in the similar Galactic binaries WR 9 (WC4+O) and WR 30a (WO5+O). WWC effects are studied in all six systems, with the following results: Very strong WWC Lührs-type effects are seen at C III 5696 in the intermediate period systems $\operatorname{Br} 22$ and WR $9(P \simeq 15 \mathrm{~d})$, while the total $\mathrm{C}$ III 5696 remains extremely weak in the very short-period systems $\mathrm{Br} 31, \mathrm{Br} 32$, WR 30a $(P \simeq 2-4 \mathrm{~d})$ and the intermediate period system AB $8(P=16.6 \mathrm{~d})$ in the SMC. Remarkably, the underlying WR subtypes in $\mathrm{Br} 22$ and WR 9 change from WC6 and WC5, respectively, to WC4 in both cases, due to the previously unrecognized confusion of the key classification $\mathrm{C}$ III 5696 line from the WR wind with the WWC source. This makes all the WC types in the LMC identical: $W C 4$. One other (single) WC/WO star in the LMC is WO4, i.e., very similar to WC4 with enhancement of O-lines, while the SMC star (AB 8) is $\mathrm{WO} 4+\mathrm{O}$.

Other strong lines in all six WCE/WO binaries show WWC effects. The clearest case is for the doublet C IV 5802/12, where the WWC Lührs' effects are seen despite the (nevertheless relatively small) smearing effect of the line doublet. However, formal fits to the Lührs' model profiles are not as satisfactory for CIV or for CIII, as in the case of WR 42 and WR 79. This may be due to the (necessarily) somewhat inferior quality of the MC data, combined with degeneracies in fitting.

Of special interest are WWC effects in elliptical orbits. In particular, three such WR+O systems have been studied so far in the context of WWC excess emission. The advantage is, despite the increase in complexity, that one can in principle probe the wind interactions at different separations in the same system. In the very long period $(P=7.94 \mathrm{yr}$ ) system WR 140 (Williams et al. 1987, $1990)$, the eccentricity is so large $(e=0.85)$ that one sees WWC line excess emission only close to periastron passage (Hervieux 1995), although heating effects may also play a role (Marchenko et al. $1999 \mathrm{~b}$ ). In the $78.5 \mathrm{~d}, e=0.33$ system $\gamma^{2}$ Velorum, the C III 5696 excess increases in strength around periastron passage and may show effects of crashing of the WR wind onto the O-star (e.g., Eversberg et al. 1999). In the $19.3 \mathrm{~d}, e=0.27$, massive, eclipsing SMC binary HD 5980, WWC effects appear to be spectacular (Moffat et al. 1998) in the more quiescent state before one of its components erupted as an LBV in 1994 . This may be due to the fact that both stars before the eruption had winds of similar strength, maximizing the effects of WWC.

\section{Conclusions}

Three main conclusions emerge from this work so far:

- WWC - LPVs appear to be universal in WR+O systems with short to moderate periods below $\approx 100 \mathrm{~d}$. They are, however, often difficult to disentangle from other effects for high inclination and very short-period systems.

- WWC line effects can be used as a tool to probe three phenomena: (1) the orbit $(i) ;(2)$ the winds of the two stars $\eta(\Theta), \delta \phi \rightarrow\left(\dot{M} v_{\infty}\right)_{\mathrm{O}} /\left(\dot{M} v_{\infty}\right)_{\mathrm{WR}}, v_{\text {orb }} / v_{\text {wind }}$; (3) the collision process itself $\left(v_{\text {strm }}, \Delta \Theta\right)$.

- WWC effects may hinder (possibly even prevent) Roche-lobe overflow or wind accretion in most $\mathrm{WR}+\mathrm{O}$ and $\mathrm{O}+\mathrm{O}$ binaries. This is less certain in bloated 
LBV/RSG+O systems, which probably preceed most WR+O binaries, although WWC must ultimately be accounted for there.

Several obvious avenues remain to be exploited in the context of this review: - Attempt to trace the shock cone from excess WWC emission in lines of different ionization potential, thus formed at different places along the shock cone.

- Explore the expected rich line spectrum and its phase-dependent variation in the far-UV and X-ray domains. The latter will arise closest to the extremely hot bow shock head.

- Attempt to predict the WWC line excess spectrum on the basis of hydrodynamical + radiative models of the WWC process, allowing for the clumpy nature of the winds even before WWC (e.g., Walder, these Proceedings).

- Resolve the WWC zones directly using stellar interferometry. Although not in the true spirit of this spectroscopic review, this will be the ultimate way to explore WWCs, thus testing all the spectral work that came before it. Even for the closest system, $\gamma^{2}$ Velorum at $d=258 \mathrm{pc}$ (van der Hucht et al. 1997), the mean orbital separation is $\sim 5$ mas, so one will need sub-mas resolution to resolve the WWC zone. This may be possible from the ground in the next few years, but more likely from space in some 10 years (using SIM, GAIA).

Acknovsledgments. I am grateful for financial aid to NSERC (Canada) and FCAR (Québec). I acknowledge my main collaborators on this project, Sergey Marchenko, Nicole St-Louis, Grant Hill and Peter Bartzakos.

\section{References}

Antokhin, I.I., Moffat, A.F.J. 1999, in preparation

Bartzakos, P. 1998, Ph.D. Thesis, Université de Montréal

Bartzakos, P., Moffat, A.F.J., Niemela, V.S. 1999, in preparation

Bertrand, J.-F. 1995, in: K.A. van der Hucht \& P.M. Williams (eds.), Wolf-Rayet Stars: Binaries, Colliding Winds, Evolution, Proc. IAU Symp. No. 163 (Dordrecht: Kluwer), p. 248

Cantó, J., Raga, A.C., Wilkin, F.P. 1996, ApJ 469, 729

Damineli, A., Conti, P.S., Lopes, D.F. 1997, New Astronomy 2, 107

Dougherty, S.M., Williams, P.M., van der Hucht, K.A., et al. 1996, MNRAS 280, 963

Eichler, D., Usov, V. 1993, ApJ 402, 271

Eversberg, T., Lépine, S., Moffat, A.F.J. 1998, ApJ 494, 799

Eversberg, T., Moffat, A.F.J., Marchenko, S.V. 1999, submitted

Foellmi, C., et al. 1999, in preparation

Gayley, K.G., Owocki, S.P., Cranmer, S.R. 1997, ApJ 475, 786

Gies, D.R. 1996, RevMexAA-CS 5, 31

Hervieux, Y. 1995, in: K.A. van der Hucht \& P.M. Williams (eds.), Wolf-Rayet Stars: Binaries, Colliding Winds, Evolution, Proc. IAU Symp. No. 163 (Dordrecht: Kluwer), p. 460

Hill, G.M., et al. 1999a,b, in preparation

van der Hucht, K.A., Schrijver, H., Stenholm, B. et al. 1997, New Astronomy 2, 245

Kallrath, J. 1991, MNRAS 248, 653

Koenigsberger, G., Auer, L.H. 1985, ApJ 297, 255 
Lamontagne, R., Moffat, A.F.J., Drissen, L., et al. 1996, AJ 112, 2227

Lépine, S., Moffat, A.F.J. 1999, ApJ 514, 909

Lewis, D., Moffat, A.F.J., Matthews, J.M., et al. 1993, ApJ 405, 312

Lührs, S. 1997, PASP 109, 504

Lührs, S., Moffat, A.F.J. 1999, in preparation

Marchenko, S.V., Moffat, A.F.J., Eenens, P.R.J., et al. 1995, ApJ 450, 811

Marchenko, S.V., Moffat, A.F.J., Lamontagne, R., et al. 1996, ApJ 461, 386

Marchenko, S.V., Moffat, A.F.J., Eenens, P.R.J., et al. 1997, ApJ 485, 826

Marchenko, S.V., Moffat, A.F.J., Eenens, P.R.J. 1998, PASP in press

Marchenko, S.V., Moffat, A.F.J., Grosdidier, Y. 1999a, ApJ submitted

Marchenko, S.V., et al. 1999b, in preparation

Moffat, A.F.J., Seggewiss, W. 1977, A\&A 54, 607

Moffat, A.F.J. 1996, RevMexAA-CS 5, 38

Moffat, A.F.J. 1998, ApSS 260, 225

Moffat, A.F.J., Marchenko, S.V., Bartzakos, P. et al. 1998, ApJ 497, 896

Moffat, A.F.J., et al. 1999, in preparation

Niemela, V.S., Barba, R.H., Shara, M.M. 1995, in: K.A. van der Hucht \& P.M. Williams (eds.), Wolf-Rayet Stars: Binaries, Colliding Winds, Evolution, Proc. IAU Symp. No. 163 (Dordrecht: Kluwer), p. 245

Rauw, G., Vreux, J.-M., Gosset, E., et al. 1996, A\&A 306, 771

Setia Gunawan, D.Y.A., van der Hucht, K.A., Williams, P.M., et al. 1995, in: K.A. van der Hucht \& P.M. Williams (eds.), Wolf-Rayet Stars: Binaries, Colliding Winds, Evolution, Proc. IAU Symp. No. 163 (Dordrecht: Kluwer), p. 466

Shore, S.N., Brown, D.N. 1988, ApJ 334, 1021

Stevens, I.R., Blondin, J.M., Pollock, A.M.T. 1992, ApJ 386, 265

Stevens, I.R., Howarth, I.D. 1999, MNRAS 302, 549

St-Louis, N., Moffat, A.F.J., Drissen, L., et al. 1988, ApJ 330, 286

St-Louis, N., Willis, A.J., Stevens, I.R. 1993, ApJ 415, 298

St-Louis, N. 1995, in: K.A. van der Hucht \& P.M. Williams (eds.), Wolf-Rayet Stars: Binaries, Colliding Winds, Evolution, Proc. IAU Symp. No. 163 (Dordrecht: Kluwer), p. 388

St-Louis, N., Hill, G., Moffat, A.F.J., et al. 1996, in: J.-M. Vreux, A. Detal, D. FraipontCaro, E. Gosset \& G. Rauw (eds.), Wolf-Rayet Stars in the Framework of Stellar Evolution, Proc. $33^{r d}$ Liège Int. Astroph. Coll. (Liège: Univ. of Liège), p. 331

Veen, P.M., van Genderen, A.M., Verheijen, M.A.W., van der Hucht, K.A., et al. 1995, in: K.A. van der Hucht \& P.M. Williams (eds.), Wolf-Rayet Stars: Binaries, Colliding Winds, Evolution, Proc. IAU Symp. No. 163 (Dordrecht: Kluwer), p. 243

Wallerstein, G., Wilson, L.A., Salzer, J., Brugel, E. 1984, A\&A 133, 137

Williams, P.M., van der Hucht, K.A., van der Woerd, et al. 1987 in: H. Lamers \& C.W.H. de Loore (eds.), Instabilities in Luminous Early Type Stars, Proc. of a Workshop in honour of Cornelis de Jager (Dordrecht: Reidel), p. 221

Williams P.M., van der Hucht, K.A., Pollock, A.M.T., et al. 1990, MNRAS 243, 662

Williams, P.M., Dougherty, S.M., Davis, R.J., van der Hucht, K.A., et al. 1997, MNRAS 289,10 


\section{Discussion}

Cantó: We have obtained an exact analytic solution (see ApJ 469, 729, 1996) for the shape, mass surface density and velocity along the shell that is formed in the collision of two isotropic stellar winds. The solution is very simple and can be used to obtain the observational characteristics of such interaction. The energetics and momentum distributions of the bow-shocks are given in: B. Reipurth \& L. Bertout (eds), HerbigHaro Flows and the Birth of Low Mass Stars, p. 343.

Owocki: Are any of the systems you've discussed close-enough in separation for radiative braking effects to be important?

Moffat: Almost certainly, but we cannot separate a priori a higher $\eta$ system with no braking from a lower $\eta$ system with braking. We need more information to do it, e.g., $v_{\infty}$ and $\dot{M}$ of both stars obtained from other considerations.

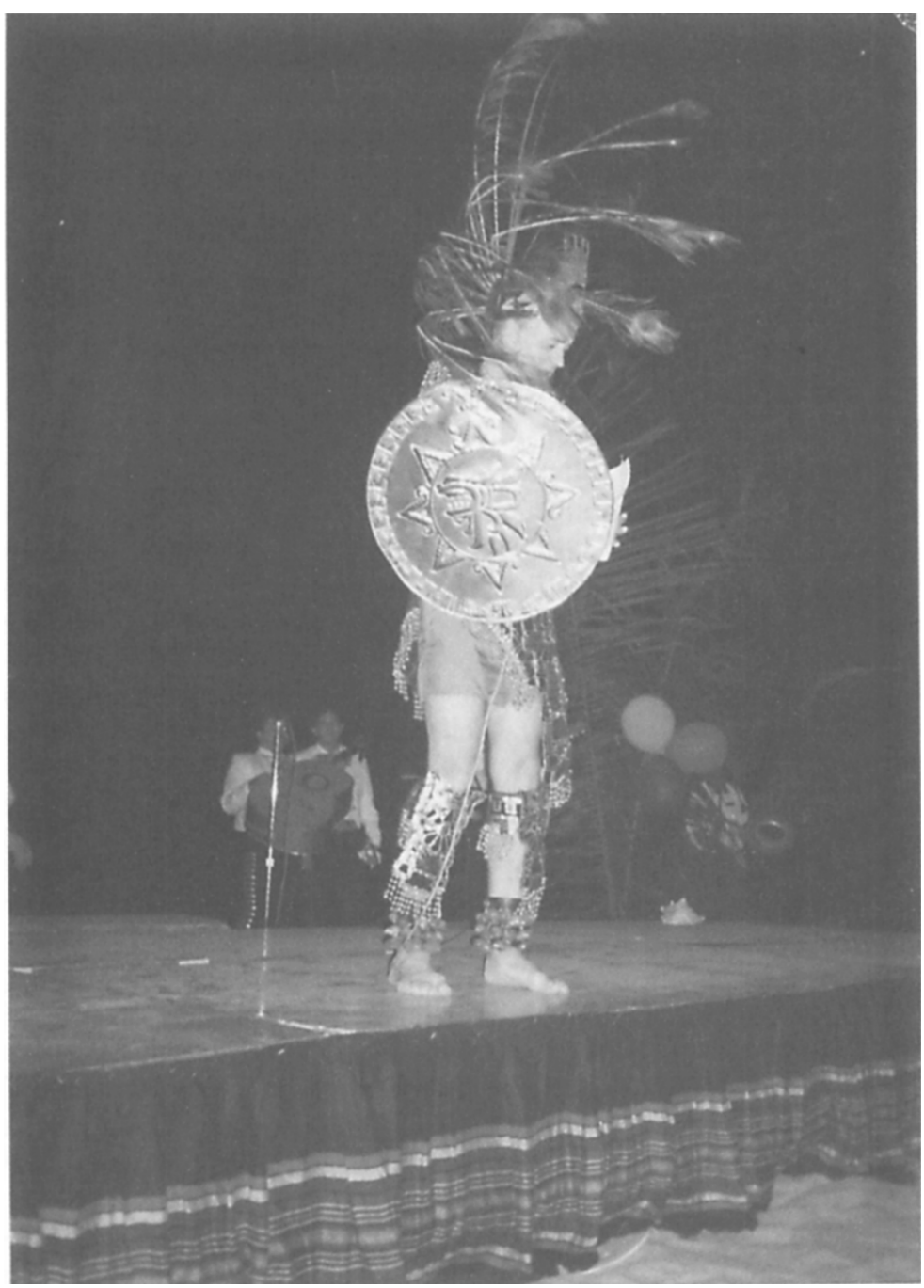

